\title{
Avaliação da infestação de insetos-praga associados à batata (Solanum tuberosum L.) sob efeito de nutrientes nitrogenados e potássicos e teores acumulados de aminoácidos livres nas cultivares Achat e Monalisa
}

\author{
Edson Henrique de Azeredo ${ }^{1}$ \\ Paulo Cesar Rodrigues Cassino ${ }^{2}$ \\ Eduardo Lima ${ }^{3}$
}

\begin{abstract}
Evaluation of insect-pest infestation associated to potato (Solanum tuberosum L.) under effect of nitrogen and potassium fertilizers and the accumulated amount of free aminoacids in Achat and Monalisa cultivars. The objective of this work was to evaluate the occurence of insect-pests on potato plants influenced by dosages of nitrogen and potassium accumulated in plant organs. A total of 169 plants of the Achat and Monalisa cultivars were evaluated to determine the presence-absence of Diabrotica speciosa Germar, 1824 and Agrotis ipsilon Hüfnagel, 1767. The experiment was carried out and executed at the Universidade Federal Fluminense, and the delineation was complete randomized block design, with four replication and nine treatments, using three fertilization level $(0 ; 75$ and $150 \mathrm{Kg} / \mathrm{ha})$ with $\mathrm{N}$-urea $+\mathrm{KCl}$. The aminoacid levels were adjusted by the Leucine standard-curve $(\mu \mathrm{g} / \mathrm{l})$, using the Ninhydrin method, at $570 \mathrm{~nm}$. The results showed that the tubercles of Monalisa accumulated high free aminoacid levels with 7,95\% in the treatment $\mathrm{N}_{1} \mathrm{~K}_{2}$ and $7,75 \%$ in the $\mathrm{N}_{2} \mathrm{~K}_{1}$. These treatments, induced the infestation by D. speciosa larvae in $27,03 \%$, when the aminoacid level was $2,01 \pm$ $0,58 \%(\mathrm{X} \pm \mathrm{EP}$ ), with probability of $0,0196<\mathrm{P}<0,05$. The studied cultivars showed a diferential behavior, especially, in relationship to the $\mathrm{N}$ and $\mathrm{K}$ metabolism, resulting in the accumulation of free aminoacids, and consequently in the pest infestation increment. The results indicated that the aminoacid level increasing affects the tubercles production, mainly in the Monalisa cultivar, in winter cropping with long dry periods.
\end{abstract}

KEYwords. Diabrotica speciosa; Agrotis ipsilon; damage level; monitoring; plant nutrition.

\section{INTRODUÇÃO}

Na cultura da batata, Solanum tuberosum L. (Solanaceae) ocorreram alguns insetos-praga que causam danos, quer pela sua ação direta, sugando a seiva ou pela mastigação da área foliar, depreciando os tubérculos para fins comerciais. No caso da batata, por se tratar de uma planta de produção subterrânea, ocorre, na fase de tuberização, o ataque esporádico de pragas do solo,como as larvas-alfinete da vaquinha, Diabrotica speciosa Germar,1824 (Coleoptera, Chrysomelidae). A lagarta rosca Agrotis ipsilon Hüfnagel,1767 (Lepidoptera, Noctuidae) é outra praga importante (Lima \& RACCA FiLHO 1996; NAKANO et al. 1981).

As causas do aparecimento de novas pragas estão associadas à dependência estreita entre qualidade nutricional da planta (CHABOussou 1987). BethKeR et al. (1987) afirmaram que altos níveis de nitrogênio proporcionam aumento no número de pupas e adultos de Liriomyza trifolli em tomateiro. Também para VAN EMDEN (1966), que estudou a relação entre inseto e planta hospedeira em diferentes níveis de $\mathrm{N}$ e $\mathrm{K}$, o nitrogênio sob forma amoniacal, aumenta o conteúdo de aminoácidos nas folhas e, portanto, sensibiliza a planta às pragas e doenças. LARA (1972), aponta alguns mecanismos de seleção de hospedeiros pelos insetos que, entre os sete princípios básicos, a seleção é feita em resposta às substâncias nutritivas ou traços, tanto quanto as substâncias secundárias.

FERRI (1979) considerou os aminoácidos protéicos substâncias livres que desempenham funções específicas na economia bioquímica da célula vegetal, sendo responsáveis pela síntese da proteína. A concentração de aminoácido, segundo HoffmAnn \& SAmish (1969), constitui o melhor critério para a determinação do estado nutricional do K nas plantas.

\footnotetext{
1. Colégio Agrícola "Nilo Peçanha”, Universidade Federal Fluminense. 27197-000 Pinheiral - RJ, Brasil.

Endereço eletrônico: edsonhenrique.azeredo @bol.com.br

2. Centro Integrado de Manejo de Pragas, Departamento de Entomologia e Fitopatologia, Instituto de Biologia, Universidade Federal Rural do Rio de Janeiro. 23890-000 Seropédica - RJ, Brasil.

3. Departamento de Solos, Instituto de Agronomia, Universidade Federal Rural do Rio de Janeiro. 23890-000 Seropédica - RJ, Brasil.
} 
Segundo Chaboussou (1969) a sanidade das plantas está intimamente ligada à qualidade de seu habitat, e se este lhe permite uma nutrição equilibrada portará resistência a fatores diversos, não permitindo danos acentuados de pragas, nem o desenvolvimento de doenças e manifestação de viroses. Esse mesmo auto, em trabalho realizado em 1972, constatou que uma planta ou órgão vegetal encontrar-se-á mais vulnerável ao ataque de pragas ou doenças na medida em que os teores de substâncias solúveis nutricionais corresponda às exigências tróficas do parasita, necessitando encontrar na planta hospedeira alimento solúvel em forma de aminoácidos livres, açúcares reduzidos, isto é, ainda não incorporados em macromoléculas insolúveis. A esta dependência estreita entre as qualidades nutricionais da planta e seu parasita, CHABoussou (1972) chamou de trofobiose.

O objetivo deste trabalho foi avaliar a ocorrência de insetos-praga associados à cultura da batata, influenciada por nutrientes nitrogenados e potássicos e a teores de aminoácidos livres, na região Sulfluminense.

\section{MATERIALE MÉTODOS}

A pesquisa foi realizada na área do campus da Universidade Federal Fluminense, em Pinheiral, RJ, localizada em uma região de clima subúmido de temperatura média de $23,5^{\circ} \mathrm{C}$, latitude $44^{\circ} 04^{\prime} 4,9^{\prime \prime} \mathrm{W}$, longitude $22^{\circ} 29^{\prime} 2,9^{\prime \prime} \mathrm{S}$ e altitude média de $473 \mathrm{~m}$. O solo do local do experimento, para efeito de análise, foi dividido estrategicamente em Amostra 1, 2 e 3 (início, meio e fundos da área), tendo em vista a avaliação dos efeitos tróficos de $\mathrm{N}+\mathrm{K}$ sobre a dinâmica populacional de $D$. speciosa e $A$. ipsilon. Assim, determinou-se:

\section{Amostra 1:}

textura média; $\mathrm{pH}_{\left(\mathrm{H}_{2} \mathrm{O}\right)}=5,4 ; \mathrm{Al}_{(\text {meq./100 ml }}^{3+}=0,0$; $\mathrm{Ca}_{(\text {meq. } / 100 \mathrm{ml})}^{2+}=4,0 ; \mathrm{Mg}_{(\text {meq. } / 100 \mathrm{ml})}^{2+}=0,6 ; \mathrm{P}_{(\mathrm{ppm})}=570 ;$ $\mathrm{K}_{(\mathrm{ppm})}=125 ; \mathrm{C}_{(\%)}=1,02 ; \mathrm{e}, \mathrm{MO}_{(\%)}=1,7$

\section{Amostra 2:}

textura arenosa; $\mathrm{pH}_{\left(\mathrm{H}_{2} \mathrm{O}\right)}=6,2 ; \mathrm{Al}_{(\text {meq. } / 100 \mathrm{ml})}^{3+}=0,0$; $\mathrm{Ca}_{(\text {meq. } / 100 \mathrm{ml})}^{2+}=3,7 ; \mathrm{Mg}_{(\text {meq. } / 100 \mathrm{ml})}^{2+}=1,3 ; \mathrm{P}_{(\mathrm{ppm})}=234 ;$ $\mathrm{K}_{(\mathrm{ppm})}=155 ; \mathrm{C}_{(\%)}=1,11 ; \mathrm{e}, \mathrm{MO}_{(\%)}=1,9$

\section{Amostra 3:}

textura arenosa; $\mathrm{pH}_{\left(\mathrm{H}_{2} \mathrm{O}\right)}=5,8 ; \mathrm{Al}_{(\text {meq./100 ml })}^{3+}=0,0$; $\mathrm{Ca}_{(\text {meq. } / 100 \mathrm{ml})}^{2+}=2,5 ; \mathrm{Mg}_{(\text {meq. } / 100 \mathrm{ml})}^{2+}=0,7 ; \mathrm{P}_{(\mathrm{ppm})}=302$; $\mathrm{K}_{(\mathrm{ppm})}=135 ; \mathrm{C}_{(\%)}=1,16 ; \mathrm{e}, \mathrm{MO}_{(\%)}=2,00$
Os dados foram coletados usando duas cultivares: Achat (procedência Alemã) e Monalisa (Holandesa), cujos tubérculossemente foram adquiridos da Fazenda Experimental de Mariada-Fé, Minas Gerais. O período de monitoramento foi de 6 de maio a 2 de agosto de 1996. As leituras foram feitas em um talhão experimental de $350 \mathrm{~m}^{2}$. O delineamento experimental foi em blocos ao acaso com esquema fatorial e parcelas subdivididas, com quatro repetições e nove tratamentos, empregando-se três níveis de adubação $\mathrm{N}+\mathrm{K}(0 ; 75$ e $150 \mathrm{~kg} /$ ha de N-uréia + cloreto de potássio) e 96 gramas de esterco de ave. Cada bloco consistia de três parcelas com nove linhas de 15,6 m de comprimento, tendo 13 plantas em cada subparcela.

Os tratamentos foram aplicados em três épocas: aos 15 dias antecedendo o plantio (juntamente à única parcela de matéria orgânica); em cobertura, aos 30 e 50 dias por ocasião da primeira e segunda pré-amontoas, no início e tuberização plena. Os tratos culturais empregados consistiram na limpeza manual entre as plantas na leira, deixando as entrelinhas com a vegetação rasteira, sendo que plantas-teste localizavam-se $30 \mathrm{~cm}$ acima. O sistema de irrigação foi por infiltração, distribuindo-se a lâmina d'água uma vez por semana até os 30 primeiros dias do plantio; a partir daí até o 50o dia do inicío do estádio de florescimento ao da tuberização, intensificou-se a dotação hídrica (duas vezes/semana), reduzindo-se a uma/semana, no estádio de fenologia de abscisão ( \pm 80-90 dias).

Quanto ao monitoramento dos insetos-praga, avaliou-se 196 plantas, tomando-se leituras de amostragens na parte superior, inferior e tubérculos (avaliados na colheita, observado o intervalo amostral $=72$ ), durante 13 semanas, geralmente no quadrante horário entre 10 e 15 horas. Empregou-se a metodologia preconizada por CAssino et al. (1983), modificada para a expressão, $\mathrm{n}=\left[(\mathrm{X} / 2)^{1 / 2}+(\mathrm{Y})^{1 / 2}\right]^{1 / 2}$ por AzEREDO (1998) em função do número de plantas por hectare e número de plantas da área do talhão pesquisado, semanalmente. Os teores acumulados de aminoácidos em folha verde, folha senescente, folha em abscisão, haste, tubérculo e planta total, foram coletados aos 75 dias após o plantio. Para isso, retirou-se duas plantas úteis, por meio de $(\mathrm{Y})^{1 / 2}$, em cada subparcela. As partes destinadas à análise química de aminoácidos livres, foram conservadas em etanol a $80 \%$, num período de seis meses, para a extração.

As leituras dos teores de aminoácidos livres foram realizadas em espectrofotômetro, "Spectronic 21-D", do Laboratório do Centro Integrado de Manejo de Pragas (CIMP), UFRRJ, através da avaliação das amostras pela curva padrão Leucina $(\mu \mathrm{g} / \mathrm{l}) \mathrm{de}$ $\mathrm{N}$-amino pelo método da Ninhydrina a $570 \mathrm{~nm}$, de Yemm \& Cocking (1955). Para análise estatística, efetuou-se a transformação dos dados (\%) de aminoácidos livres pela raiz (variável +2$)$ e, através da análise de variância, teste $\mathrm{F}$, as médias comparadas entre si, pelo teste de Tukey a 5\%. Em relação aos insetos-praga, as freqüências de campo foram transformadas para graus de infestação (\%) médios por arc $\operatorname{sen} \sqrt{\% / 100}$, e a significância, através da correlação linear simples de Pearson, "T", $\mathrm{P}<0,05$. 
Tabela I. Teores acumulados de aminoácidos livres (\%) em planta de batata, Solanum tuberosum L., cultivares Achat e Monalisa, destinados à avaliação do grau de infestação de insetos-praga, em relação às dosagens de N e K (0; 75 e 150 kg/ha), no período de maio a agosto de 1996 . Pinheiral/ RJ.

\begin{tabular}{|c|c|c|c|c|c|c|c|c|c|c|c|c|}
\hline \multirow[t]{2}{*}{$\begin{array}{c}\text { Tratamento }^{1,2} \\
\text { (BLOCOS) }\end{array}$} & \multicolumn{2}{|c|}{$\begin{array}{c}\text { Folha verde } \\
(\%)\end{array}$} & \multicolumn{2}{|c|}{$\begin{array}{c}\text { Folha em } \\
\text { senescência } \\
(\%)\end{array}$} & \multicolumn{2}{|c|}{$\begin{array}{c}\text { Folha em } \\
\text { abscisão } \\
(\%)\end{array}$} & \multicolumn{2}{|c|}{$\begin{array}{l}\text { Haste } \\
(\%)\end{array}$} & \multicolumn{2}{|c|}{$\begin{array}{c}\text { Tubérculos } \\
(\%)\end{array}$} & \multicolumn{2}{|c|}{$\begin{array}{l}\text { Planta total } \\
(\%)\end{array}$} \\
\hline & Ach. & Mon. & Ach. & Mon. & Ach. & Mon. & Ach. & Mon. & Ach. & Mon. & Ach. & Mon. \\
\hline \multirow[t]{2}{*}{$\mathrm{N}_{0} \mathrm{~K}_{0}$} & 3,24 & 1,50 & 3,81 & 4,07 & 1,20 & 0,85 & 2,84 & 4,06 & 1,43 & 7,60 & 2,26 & 7,74 \\
\hline & IV & II & IV & IV & I & II & III & IV & I & III & II & IV \\
\hline \multirow[t]{2}{*}{$\mathrm{N}_{0} \mathrm{~K}_{1}$} & 3,24 & 5,61 & 1,86 & 3,24 & 0,63 & 1,36 & 3,03 & 0,86 & 1,52 & 2,27 & 3,47 & 7,52 \\
\hline & IV & IV & IV & III & I & III & II & II & III & II & III & I \\
\hline \multirow[t]{2}{*}{$\mathrm{N}_{0} \mathrm{~K}_{2}$} & 2,80 & 2,85 & 1,59 & 3,74 & 3,98 & 1,16 & 1,44 & 1,51 & 3,24 & 5,07 & 3,92 & 2,47 \\
\hline & III & IV & III & I & IV & III & III & III & IV & I & IV & III \\
\hline \multirow[t]{2}{*}{$\mathrm{N}_{1} \mathrm{~K}_{0}$} & 3,84 & 2,13 & 2,18 & 1,51 & 3,18 & 0,84 & 3,75 & 2,22 & 3,69 & 7,86 & 2,10 & 5,48 \\
\hline & III & II & III & II & I & III & IV & III & II & I & I & II \\
\hline \multirow[t]{2}{*}{$\mathrm{N}_{1} \mathrm{~K}_{1}$} & 3,87 & 3,12 & 3,79 & 3,36 & 0,60 & 1,68 & 2,46 & 2,07 & 3,99 & 3,33 & 3,42 & 3,07 \\
\hline & IV & III & II & II & II & I & IV & I & I & I & IV & II \\
\hline \multirow[t]{2}{*}{$\mathrm{N}_{1} \mathrm{~K}_{2}$} & 2,93 & 5,91 & 2,51 & 2,10 & 0,64 & 2,02 & 3,53 & 4,77 & 1,86 & 7,95 & 1,69 & 4,61 \\
\hline & IV & III & III & III & III & IV & III & III & IV & II & IV & III \\
\hline \multirow[t]{2}{*}{$\mathrm{N}_{2} \mathrm{~K}_{0}$} & 1,46 & 1,42 & 1,88 & 1,85 & 0,84 & 1,20 & 0,82 & 3,02 & 3,77 & 6,81 & 1,91 & 7,88 \\
\hline & II & I & IV & I & IV & II & I & II & II & I & I & I \\
\hline \multirow[t]{2}{*}{$\mathrm{N}_{2} \mathrm{~K}_{1}$} & 2,88 & 2,65 & 3,73 & 4,31 & 1,20 & 1,81 & 1,52 & 6,79 & 1,28 & 7,75 & 1,32 & 7,44 \\
\hline & IV & III & IV & IV & III & II & IV & III & I & III & II & III \\
\hline \multirow[t]{2}{*}{$\mathrm{N}_{2} \mathrm{~K}_{2}$} & 1,48 & 4,05 & 1,56 & 3,14 & 3,80 & 1,54 & 1,20 & 1,48 & 3,83 & 3,66 & 2,34 & 2,45 \\
\hline & III & III & II & III & III & I & III & III & II & II & II & III \\
\hline
\end{tabular}

1. Análises químicas realizadas pelo Laboratório do Centro Integrado de Manejo de Pragas "Cincinnato Rory Gonçalves", da Universidade Federal Rural do Rio de Janeiro.

2. Teores acumulados de aminoácidos e respectivos blocos de onde, aos 75 dias após o plantio, foram retiradas as plantas amostradas.

\section{RESULTADOS EDISCUSSÃO}

Os resultados da análise química nos órgãos de batata amostrados e de interesse da análise fenológico-fitossanitária, demonstraram: na cultivar Monalisa houve um acúmulo significativo de aminoácidos livres no órgão folha verde (Tabelas I e II; Fig. 1) entre os tratamentos $\mathrm{N}_{0} \mathrm{~K}_{0}$ e $\mathrm{N}_{0} \mathrm{~K}_{1}$, indo de 1,50 para $5,61 \%$, especificamente; reduzindo-se a $2,85 \%$, assim que o potássio recebeu dosagem máxima $\left(150 \mathrm{~kg}\right.$ de $\left.\mathrm{K}_{2} \mathrm{O} / \mathrm{ha}\right)$. Em relação ao tratamento $\mathrm{N}_{2} \mathrm{~K}_{2}$, a velocidade de concentração atingiu $1,48 \%$, diferindo da cv. Achat que mostrou teor máximo de $4,05 \%$.

Segundo Labrousse (1932), qualquer adubação que deixe a planta em condição fisiológica ótima, confere-lhe o máximo de resistência. Portanto, tanto o excesso como a carência de um ou diversos elementos, que rompem o equilíbrio fisiológico normal da planta, são capazes de diminuir sua resistência natural. Na relação entre a planta e seu parasita, antes de tudo, influem os fatores nutricionais. Daí a importância do "condicionamento" da planta pela natureza do solo e pela fertilização "corretiva" (Roubine \& ARTTSICHOVSKAIA 1960).

Ao se isolar os nutrientes, na cv. Monalisa, observou-se que de $\mathrm{K}_{1}$ para $\mathrm{K}_{2}$, ou seja, de 75 para $150 \mathrm{~kg} . \mathrm{K}_{2} \mathrm{O} / \mathrm{ha}$, houve um incremento no teor de aminoácidos no estádio vegetativo inicial da planta de 3,79 para 4,27\% (Tabela II), quando comparado à outra cultivar que registrou uma redução nesse composto. Entretanto, avaliando-se o grau de infestação (Tabela III), verificou-se que a dosagem máxima de potássio $(150 \mathrm{~kg}$ de 
Tabela II. Teores acumulados de aminoácidos livres nos diferentes órgãos amostrados e em relação às dosagens de $\mathrm{Ne} \mathrm{K}$ ( $0 ; 75$ e 150 kg/ha), isolados em planta da batata, Solanum tuberosum L., cultivares Achat e Monalisa, no período de 06 de maio a 02 de agosto de 1996, no município de Pinheiral, RJ.

\begin{tabular}{|c|c|c|c|c|c|c|c|c|c|c|c|c|}
\hline \multirow[t]{2}{*}{ Variável } & \multicolumn{6}{|c|}{ Achat } & \multicolumn{6}{|c|}{ Monalisa } \\
\hline & $\mathrm{N}_{0}$ & $\mathrm{~N}_{1}$ & $\mathrm{~N}_{2}$ & $\mathrm{~K}_{0}$ & $\mathrm{~K}_{1}$ & $\mathrm{~K}_{2}$ & $\mathrm{~N}_{0}$ & $\mathrm{~N}_{1}$ & $\mathrm{~N}_{2}$ & $\mathrm{~K}_{0}$ & $\mathrm{~K}_{1}$ & $\mathrm{~K}_{2}$ \\
\hline Folha verde & 3,09 & 3,54 & 1,94 & 2,84 & 3,33 & 2,40 & 3,32 & 3,72 & 2,70 & 1,68 & 3,79 & 4,27 \\
\hline $\begin{array}{c}\text { Folha } \\
\text { senescente }\end{array}$ & 2,42 & 2,82 & 3,01 & 2,62 & 3,12 & 1,88 & 3,68 & 2,32 & 3,10 & 2,47 & 3,63 & 2,99 \\
\hline $\begin{array}{l}\text { Folha em } \\
\text { abscisão }\end{array}$ & 1,93 & 1,47 & 1,93 & 1,74 & 0,81 & 2,80 & 1,12 & 1,51 & 1,51 & 0,96 & 1,61 & 1,57 \\
\hline Haste & 2,43 & 3,24 & 1,18 & 2,47 & 2,33 & 2,05 & 2,14 & 3,02 & 3,76 & 3,10 & 3,22 & 2,58 \\
\hline Tubérculo & 2,06 & 3,18 & 2,96 & 2,96 & 2,26 & 2,97 & 4,98 & 6,38 & 6,07 & 7,42 & 4,45 & 5,56 \\
\hline Planta total & 3,21 & 2,40 & 1,85 & 2,09 & 2,73 & 2,65 & 5,91 & 8,78 & 5,92 & 7,03 & 6,01 & 3,17 \\
\hline
\end{tabular}

$\mathrm{K}_{2} \mathrm{O} /$ ha) propiciou a prevalência de adultos de D. speciosa, principalmente em ramos do terço médio superior da $S$. tuberosum. Desse modo, as plantas da cv. Monalisa mostraramse sensíveis ao ataque por esse crisomelídeo, ao que parece, devido ao potássio oferecido ter acarretado um aumento dos teores de aminoácidos nos vacúolos celulares, propiciando a invasão de $D$. speciosa. Este fato confirma o observado por TORREs (1995) na cultura do jiló, que é igualmente uma solanácea, onde o crescimento populacional do percevejo Corythaica cyathicollis, no ápice da planta, foi acentuado, similarmente às concentrações de aminoácidos que também aumentaram nesse órgão do vegetal.

DADD \& MitTLER (1965) estudaram os requisitos nutricionais de Myzus persicae Sulzer (Hemiptera, Aphididae). Os autores constataram que, ao se retirar os aminoácidos da dieta, a capacidade reprodutiva cai de 30 para 10 ninfas/fêmea. Esse efeito foi também observado por CARTIER (1968), que verificou uma influência definitiva das substâncias nutritivas sobre a alimentação dos pulgões Acyrthosiphon pisum e Macrosiphum euphorbiae,

Analisando-se as folhas de batata no período de senescência (Fig. 2), pode-se observar a mesma tendência em o teor de aminoácidos situar-se em concentrações elevadas na cv. Monalisa, quando 3,70\% foi a média determinada, enquanto a cv. Achat mostrou apenas 2,70\%. Conforme TоміYAмa (1963) o estado de proteólise nos tecidos da planta é consequiência de fatores como os tratamentos com agrotóxicos e fertilizantes químicos solúveis, quando propiciam a disponibilidade dos elementos convenientes ao parasita, enquanto a resistência deveria ser inerente a um ótimo de proteossíntese.
No estádio de folha em abscisão (Fig. 3), os maiores teores evidenciaram-se nas interações $\mathrm{N}_{0} \mathrm{~K}_{2}$ e $\mathrm{N}_{2} \mathrm{~K}_{2}$, com 3,98 e 3,80\%, respectivamente, na cv. Achat. Entretanto, na cv. Monalisa, o teor desse composto foi $2,02 \%$ quando as plantas foram submetidas ao tratamento $\mathrm{N}_{1} \mathrm{~K}_{2}$.

$\mathrm{O}$ efeito dos teores de aminoácidos sobre a infestação de D. speciosa e A. ipsilon pode ter sido decorrência, na cultivar Achat, de duas situações: a ausência de nitrogênio induziu ao maior grau de infestação de 18,39 e $20 \%$, respectivamente, enquato na cv. Monalisa os insetos-praga distribuíram-se em 22,22 e $25,48 \%$, porém quando se aplicou a dosagem maior (150kg.N/ha) (Tabelas III e IV). Entretanto, em relação ao potássio, o efeito sobre as pragas foi diferenciado na parte superior e nos tubérculos das plantas amostradas da cv. Monalisa. Na parte superior, a dosagem máxima $\left(\mathrm{K}_{2}=150 \mathrm{~kg}\right.$ de $\mathrm{K}_{2} \mathrm{O} / \mathrm{ha}$ ) inibiu a retomada da infestação por $D$. speciosa, na cv. Achat, não ocorrendo o mesmo com a cv. Monalisa que manteve o mesmo nível de infestação. Por outro lado, na parte inferior da planta, a ausência e o excesso de potássio, induziram a mesma infestação por A. ipsilon (em 21,90 e 21,43\%), especificamente na cv. Achat, o que não se evidenciou na outra cultivar, quando ocorreu um efeito inibitório ao nível de $70 \%$ da infestação, registrando 23,52 e 7,84\%, respectivamente, para as duas espécies de insetos (Tabelas III e IV).

No tocante ao acúmulo dos teores de aminoácidos livres, em folha em abscisão, as melhores comparações tróficas $(\mathrm{N}+$ $\mathrm{K}$ ) foram: $\mathrm{N}_{1} \mathrm{~K}_{1}$ e $\mathrm{N}_{2} \mathrm{~K}_{1}$ (média $\pm \mathrm{EP}=1,03 \pm 0,77 \%, \mathrm{~F}=3,42$; $\mathrm{P}$ $=0,05 \mathrm{e} \mathrm{CV}=17,4 \%)$. Entretanto, ao se aplicar os tratamentos $\mathrm{N}_{0} \mathrm{~K}_{0}$ (testemunha) e $\mathrm{N}_{2} \mathrm{~K}_{2}$, determinou-se os valores $1,33 \pm$ $0,68 \%, \mathrm{~F}=7,04, \mathrm{P}<0,10 \mathrm{e} \mathrm{CV}=17,4 \%$. 
Tabela III. Percentagens de infestação da vaquinha, Diabrotica speciosa, em planta de batata, Solanum tuberosum L., cultivares Achat e Monalisa, em relação aos níveis de $\mathrm{N}$ e K, isolados, no período de 06 de maio a 02 de agosto de 1996, no município de Pinheiral, RJ.

\begin{tabular}{|c|c|c|c|c|c|}
\hline \multirow{3}{*}{$\begin{array}{r}\text { Nível de } \\
\text { e K }\end{array}$} & \multirow{3}{*}{$\mathrm{N}$} & \multicolumn{4}{|c|}{ Cultivar } \\
\hline & & \multicolumn{2}{|c|}{ Achat $(\%)$} & \multicolumn{2}{|c|}{ Monalisa (\%) } \\
\hline & & Superior & Tubérculo & Superior & Tubérculo \\
\hline $\mathrm{N}_{0}$ & & 18,39 & 21,38 & 6,06 & 1,80 \\
\hline $\mathrm{N}_{1}$ & & 14,94 & 11,94 & 5,05 & 4,50 \\
\hline $\mathrm{N}_{2}$ & & 0 & 0 & 22,22 & 27,03 \\
\hline $\mathrm{K}_{0}$ & & 25,28 & 24,53 & 17,18 & 16,66 \\
\hline $\mathrm{K}_{1}$ & & 0 & 0 & 0 & 1,80 \\
\hline $\mathrm{K}_{2}$ & & 8,04 & 8,80 & 15,65 & 14,86 \\
\hline
\end{tabular}

Tabela IV. Percentagens de infestação da lagarta rosca, Agrotis ipsilon, em planta de batata, Solanum tuberosum L., cultivares Achat e Monalisa, em relação aos níveis de N e K, isolados, no período de 06 de maio a 02 de agosto de 1996, no município de Pinheiral, RJ.

\begin{tabular}{ccc}
\hline & \multicolumn{2}{c}{ Cultivar } \\
\cline { 2 - 3 } Nível de N e K & Achat $(\%)$ & Monalisa (\%) \\
\cline { 2 - 3 } $\mathrm{N}_{0}$ & Inferior & Inferior \\
$\mathrm{N}_{1}$ & 20,00 & 0 \\
$\mathrm{~N}_{2}$ & 11,43 & 7,84 \\
$\mathrm{~K}_{0}$ & 1,90 & 25,48 \\
$\mathrm{~K}_{1}$ & 21,90 & 23,52 \\
$\mathrm{~K}_{2}$ & 0 & 1,96 \\
& 21,43 & 7,84
\end{tabular}

Avaliando-se os teores de aminoácidos acumulados nas hastes (Fig. 4), observa-se no tocante à presença da A. ipsilon que, em dosagem maior, o nitrogênio oferecido de $150 \mathrm{~kg} / \mathrm{ha}$ fez a cv. Achat demonstrar um efeito genotípico (ou especificidade metabólica) ao comportamento do lepidópteropraga, enquanto na cv. Monalisa parece não possuir a mesma tendência, sensibilizando-se àquela dosagem. Embora o nitrogênio exerça funções essenciais nos vegetais, os fertilizantes químicos nitrogenados, especialmente os amoniacais, têm sido citados como causadores de efeitos drásticos, sensibilizando a pragas e doenças (GuAzzelli 1987). Entretanto, em relação ao potássio, essa mesma autora afirma que a função principal é atribuir resistência à planta.

Quanto ao tubérculo (Tabela I; Fig.5 ) observou-se que os teores de aminoácidos livres, na cv. Achat, não ultrapassaram $3,99 \%$ no tratamento $\mathrm{N}_{1} \mathrm{~K}_{1}$ o que não se percebe em Monalisa, que armazenou 7,95\% em $\mathrm{N}_{1} \mathrm{~K}_{2}$. Em relação ao registro de insetos-praga, em interação com os nutrientes $\mathrm{N}+\mathrm{K}$ e comportamento das cultivares, observou-se que na cv. Achat a ausência de nitrogênio " $\mathrm{N}_{0}$ " induziu a 21,38\% de infestação, reduzindo-se a "zero" assim que se aplicou o nível " $\mathrm{N}_{2}$ " (150 kg de N-uréia/ha) (Tabela III). Entretanto, na cv. Monalisa houve um efeito inverso, isto é, a infestação pela larva-alfinete passou de 1,80 para 27,03\%, concluindo-se que a cv. Monalisa é sensível ao nitrogênio em dosagem excessiva, de 75 para 150 $\mathrm{kg} / \mathrm{ha}$.

O incremento na infestação de $D$. speciosa possivelmente esteja associado às características genotípicas da cv. Monalisa, nessa etapa da fenologia produtiva. Observou-se que os tubérculos amostrados, neste estudo, exibiram tendência ao acometimento por larvas da D. speciosa, cujo efeito parece estar relacionado ao acúmulo de teores de aminoácidos livres, que pode ser atribuído à aplicação da dosagem maior de $\mathrm{N}$, induzindo a esse comportamento diferencial, conforme Tabela II, quando apresentou um aumento de 4,98 para $6,07 \%$ desse composto.

Quanto ao potássio, constatou-se as mesmas interações entre dosagem e efeito indutivo/inibitório à larva-alfinete em 

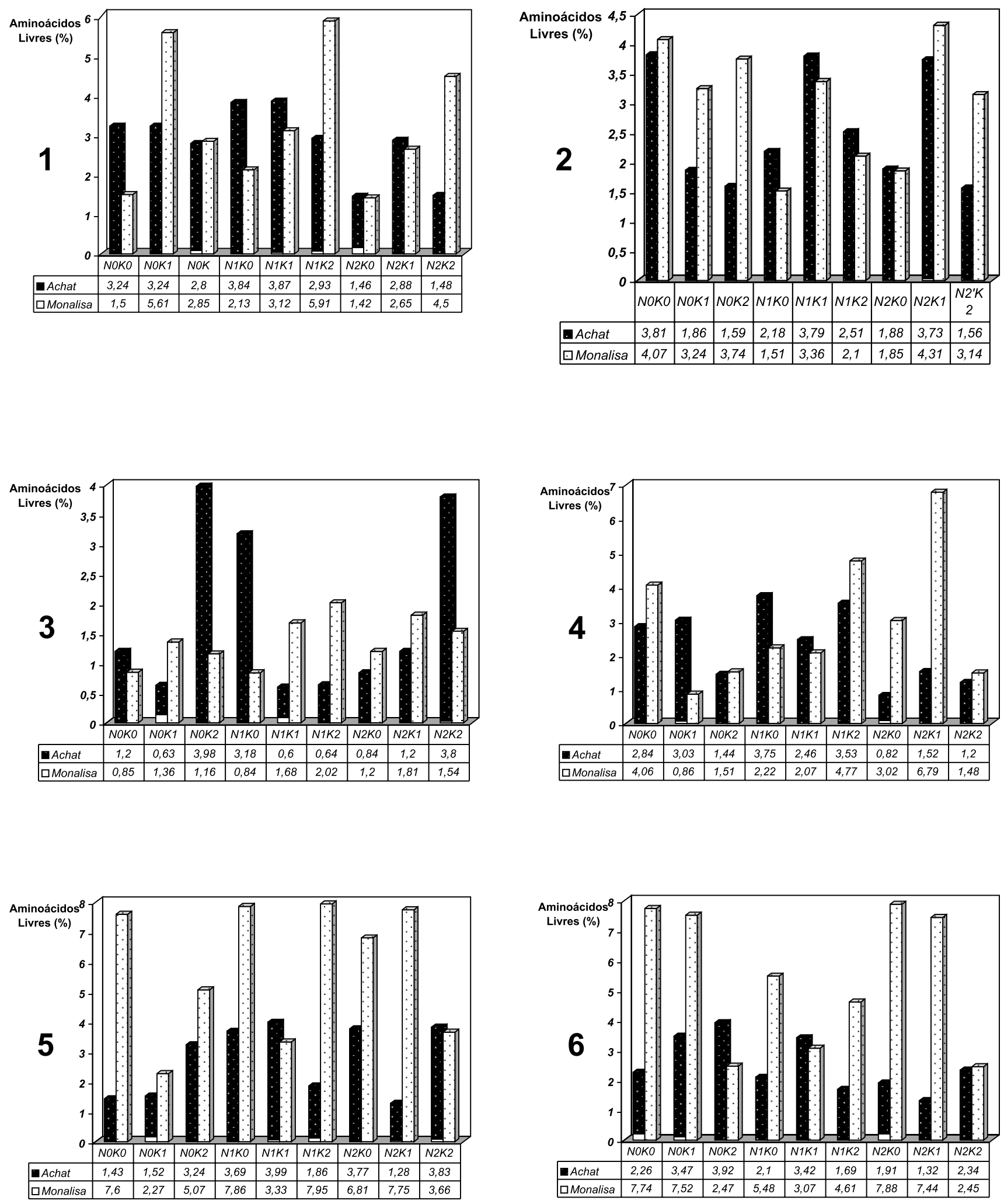

Figs. 1-6. Teores acumulados de aminoácidos livres (\%) em planta de batata, Solanum tuberosum L., cv. Achat e Monalisa, aos 75 dias após o plantio e em relação aos níveis de N e K. Pinheiral, RJ (06/07/1996). 1, em folhas verdes; 2, em folhas senescentes; 3, em folhas no período de abscisão; 4, em hastes; 5, em tubérculos; 6, na planta total. 
ambas as cultivares, quando a ausência e o excesso propiciaram a prevalência do inseto. Contrariando a afirmativa de MALAVOLTA et al. (1997), que observaram que o efeito do $\mathrm{K}$ na atividade das enzimas está relacionado com a mudança na conformação das moléculas e que é possível que uma das razões para as altas exigências potássicas seja a necessidade de concentrações elevadas no citoplasma.

Observando os efeitos de adubações potássicas sobre os teores de aminoácidos solúveis em plantas não-leguminosas, Koch \& Mengel (1972) verificaram uma redução nesses compostos em correlação com seu efeito positivo sobre a proteossíntese.

No entanto, no presente trabalho, foi constatado que o nível " $\mathrm{K}_{1}$ " (75 $\mathrm{kg}$ de $\mathrm{K}_{2} \mathrm{O} / \mathrm{ha}$ ) propiciou um efeito inibitório sobre D. speciosa. Em relação aos efeitos tróficos de N + K na variável tubérculo e após avaliadas todas as comparações possíveis, observou-se que os teores de aminoácidos acumulados em maior concentração ocorreram, respectivamente, em: $\mathrm{N}_{0} \mathrm{~K}_{1}$ e $\mathrm{N}_{2} \mathrm{~K}_{2}$ (média $\pm \mathrm{EP}=1,87 \pm 0,76 \%$, $\mathrm{F}$ $=5,61, \mathrm{P}<0,05$ e CV $=19,3 \%$ ) e $\mathrm{N}_{0} \mathrm{~K}_{1}$ e $\mathrm{N}_{2} \mathrm{~K}_{0}$ (média $\pm \mathrm{EP}=2,11$ $\pm 1,09 \%, \mathrm{~F}=6,01, \mathrm{P}<0,05 \mathrm{e} \mathrm{CV}=19,3 \%)$.

Na batata, ZAMBON et al.(1991) verificaram um aumento da população de Lyriomyza $\mathbf{s p , ~ p r i n c i p a l m e n t e ~ e m ~ d o s a g e m ~}$ elevada de nutrientes. Para CARnevalli et al. (1997) a menor população de ninfas de Thrips spp.(Thysanoptera, Thripidae) em $S$. tuberosum, ocorreu nos tratamentos com falta de N, enquanto a falta de $\mathrm{K}$, propiciou um efeito indutivo sobre a dinâmica populacional da praga. Ainda, em relação a esses insetos fitófagos, a elevação no teor de nitrogênio solúvel no floema, decorrente da aplicação de adubos nitrogenados, propicia a capacidade de reprodução de pulgões (Brevicoryne brassicae e Myzus persicae)(vAN EMDEN 1966).

Os resultados demonstraram que, em relação ao acúmulo de aminoácidos livres, as cultivares estudadas mostraram comportamento diferencial. Na cultivar Monalisa foi observada uma variação significativa, principalmente em tubérculo e planta total (Fig. 6). Esta diferença foi, possivelmente, em decorrência da sensibilidade demonstrada pela cv. Monalisa a altas temperaturas e baixos índices pluviométricos, causando duplo estresse. Provavelmente, isto tenha causado um efeito negativo durante o estádio de crescimento vegetativo, causando um desarranjo metabólico e, portanto, exibindo um distúrbio enzimático favorável ao acúmulo de teores de aminoácidos, especificamente no órgão tubérculo.

De acordo com os resultados obtidos nesse trabalho com as cultivares Achat e Monalisa, sugere-se uma observação mais detalhada no comportamento desta última, principalmente em relação à sensibilidade demonstrada a secas prolongadas, para o regime de cultivo tipo "inverno".

No presente trabalho, o comportamento da cv. Monalisa na sua performance genotípica pode ser considerado um parâmetro importante para futuros estudos sobre os efeitos tróficos nos processos inibitório/indutivo às espécies-praga, em $S$. tuberosum.

\section{REFERÊNCIAS}

Azeredo, E. H. DE. 1998. Bioecologia e influência de nutrientes $\mathbf{N}$ e $K$, sobre "insetos-praga", doenças e fenologia, no agoecossistema batatinha (Solanum tuberosum L.), no município de Pinheiral, RJ. Universidade Federal Rural do Rio de Janeiro, Seropédica, Rio de Janeiro, 313 p. (Tese de Doutorado).

Bethike, J. A..; M. P. Parrela ; J. T. Trumble \& N. C. Toscano. 1987. Effect of tomato cultivar and fertilizer regime on survival of Liriomyza trifolli (Diptera: Agromyzidae). Journal of Economic Entomology 30:200-203.

Carnevalli, P. C .; J. H. C. Lima; R. C. Andrade \& J. L. Florcovski. 1997. Diferentes tipos de adubação influindo na população de Thrips spp., em batata (Solanum tuberosum L.), p.310. In: Resumos, XVI Congresso Brasileiro de Entomologia, Salvador, 489p.

Cassino, P. C. R.; M. S. Guajara \& R. P. A. Alves. 1983. Monitoramento, estratégia básica utilizada no manejo integrado de fitoparasitos de Citrus sp., p. 843. In: Resumos, 35a Reunião Anual da SBPC, Belém.

CARTIER, J. J. 1968. Factors of host plant specifity and artificial diets. Bulletin of the Entomological Society of America 14: 18-21.

Снавоussou, F. 1969. Recherches sur les fact de pululation des acariens phytophages de la vigne à la suit des traitments pesticides du fenillage. Faculté des Sciences de l'Université de Paris, Paris, 238 p. (Thèse).

Chaboussou, F. 1972. La trofobiose et la protection de la plante. Revue des Questions Scientifiques 443 (1-2): 175-208.

DADD, R . H. \& T. E. J. MitTleR. 1965. Studies on the artificial feeding of the aphid Myzus persicae Sulzer. Some major nutritional requirements. Journal of Insect Phisiology 11:717-743.

FerRI, M. B. 1979. Fisiologia Vegetal. São Paulo, USP, 350p.

Guazelli, M. J. 1987. Plantas doentes pelo uso de agrotóxicos: a teoria da trofobiose - Francis Chaboussou (tradução). Porto Alegre, L. \& PM., 253p.

Hoffmann, M. \& R. H. SAmish. 1969. Free amino content in fruit trees organs as indicator of the nutritional status with respect to potassium. Volcani Institute of Agriculture Research:189-206.

Косн, K. \& K. Mengel .1972 . Effect of potassium nutrition on the content and the spectrum of soluble amino-coumpounds in red clover. Zeitscfr. Pfllanz 131(2): 148-154.

Labrousse, F. 1932. La fecundité du sol. Paris, Annales Agronomique, $311 \mathrm{p}$.

Lara, F. M .1991. Princípios de resistência de plantas aos insetos. São Paulo, Ícone, 336p.

Lima, A. F. \& F. Racca Filho. 1996. Manual de Pragas e Praguicidas: Receituário Agronômico. Rio de Janeiro, Universidade Rural, 818 p.

Malavolta, E.; G. C. Vitti \& S. A. de Oliveira. 1997. Avaliação do Estado Nutricional das Plantas: Princípios e Aplicações, 2a ed. Piracicaba, POTAFOS, 319 p.

NAKANo, O.; S. S. Neto \& R. A. ZucChi .1981.Entomologia Econômica.São Paulo, $31 \mathrm{p}$.

Panizzi, A. R. \& J. R. P. Parra. 1991. Ecologia Nutricional de Insetos e suas Implicações no Manejo de Pragas. São Paulo, Manole, $359 \mathrm{p}$.

Roubine, B. A .\& E. V. Artsichovskaia.1960. Biochimic et physiologie de l'immunité des plants. URSS. Ac. Science, 350p.

TomiYam, K. 1963. Physiology and biochemistry of diseases resistance of plants. Annals and Review of Phytopathology 1: 295-324.

TORRES, J. L. R. 1995. Influência da adubação nitrogenada e fatores climáticos sobre a flutuação populacional de Corythaica cyathicollis (Costa, 1864) (Hemiptera: Tingidae) em jiló (Solanum gilo Raddi) em Seropédica - RJ. Universidade Federal Rural do Rio de Janeiro, Itaguaí, Rio de Janeiro, 52 p. (Tese de Mestrado).

Van EmDen, H. F. 1966. Studies on the relations of insect and host plant III,- a comparison of the reproduction of Brevicoryne brassicae and Myzus persicae (Hemiptera:Aphididae) on brussels spout plants supllied with different rates of nitrogen and potassium. Entomologia Experimentalis et Applicata 9: 444-460. 
Yemm, E. W. \& E. C. Cocking. 1955. The determination of aminoacids by ninhydrin analyst. Biochemistry 80: 209-213.

Zambon, S.; M. H. Calafiori; N. T. Teixeira \& E. A .Cirelli .1991.Efeitos na produção e controle de Lyriomyza sp.,na batata (Solanum tuberosum) pela adubação e inseticida sistêmico, p. 345. In: Resumos, XIII Congresso Brasileiro de Entomologia, Recife. 